\title{
6.4 EXTERNALISIERUNG ALS PRINZIP IM DISKURS UM ,ENERGIEWENDE“
}

In Kapitel 3.2.2 wurde Externalisierung als Prinzip als Kriterium Vorsorgenden Wirtschaftens beschrieben und die theoretischen Parallelen zur Diskurstheorie von Laclau/Mouffe (2015) aufgezeigt. ${ }^{23}$ Die Externalisierungsstrukturen im Diskurs um „Energiewende“ werden im Folgenden zur besseren Strukturierung des Kapitels anhand von im Diskurs identifizierten hierarchisch strukturierten Dichotomien herausgearbeitet, innerhalb derer sich Abgrenzungs-/Abspaltungs-, Abwertungs- und Aneignungsprozesse zeigen.

\section{Kultur/Natur}

Becker/Jahn (2006) bezeichnen die Natur-Kultur-Unterscheidung als geschlechtlich kodierte ,zentrale Ordnungskategorie des hegemonialen westlichen Denkens“ (Becker/Jahn 2006: 227). ${ }^{24}$ Unabhängig der Ergebnisse der vorliegenden Arbeit bieten erneuerbare Energien zweifellos Potenziale für die Untergrabung dieser Dichotomie. So findet mit den erneuerbaren Energien eine graduelle Vereinigung von Schutz und Nutzung sowie Erhalten und Gestalten von Natur statt: bspw. durch die Vermittlung der Vermeidung von Ausbeutung fossiler Ressourcen sowie von Treibhausgasemissionen mit der Nutzung von Naturleistungen für die Energieproduktion.

Die vorliegende Analyse legt bisher allerdings den Rückschluss nahe, dass der „Energiewende“-Diskurs im Bundestag nicht vermittelnd, sondern verwertungsorientiert ausgerichtet ist und mit weiteren Ausschlussprozessen einhergeht.

So wird Natur in den Debatten kaum artikuliert - wenn, dann wird sie passiv, als etwas zu Schützendes konstituiert, während ihre ständige Nutzung abgespalten und ausgeblendet wird. Gleichzeitig wird Natur auf Klima reduziert, wodurch Klimaschutz als einziges umweltpolitisches Ziel politischer Praktiken im Rahmen erneuerbarer Energien und „Energiewende“ erscheint. Dies wird bspw. in der Kollokationsanalyse deutlich, in der „Naturschutz“ lediglich in der 14. Legislaturperiode als Knotenpunkt der Äquivalenzkette um „Energiewende“ auftaucht, während sich in der 15., 17. und 18. Legislaturperiode die ökologische Dimension auf „Klimaschutz“ reduziert. Darunter werden aber lediglich naturwissenschaftlich messbare und ökono-

23 Ebenso wie Biesecker/von Winterfeld (2014) für die Beschreibung von Externalisierungsstrukturen gehen auch Laclau/Mouffe (2015) von dualistischen Trennungsstrukturen aus, die für Identitäten konstitutiv und hierarchisch strukturiert seien. „Externalisierung als Prinzip bedeutet zunächst, dass etwas zum Außen gemacht werden muss, das dann als Abgespaltenes angeeignet werden kann. [...] Es findet sich überall dort, wo Menschen als nicht Zugehörige be- und vernutzt und wo Natur als ,frei` verfügbare, dem Wertvollen äuBere Ressource verbraucht wird“ (Biesecker/von Winterfeld 2014: 11).

24 Mit der Natur/Kultur Dichotomie einher gehen weitere hierarchisch strukturierte Dichotomien, bspw. Schutz/Nutzung, Statik/Dynamik, Erhalten/Gestalten oder Bewerten/Verwerten. 
misch handelbare Treibhausgaskonzentrationen einbezogen - weitere Kollokationen mit Bezug auf Natur kommen nicht vor ${ }^{25}$ (vgl. Kapitel 5.1.2).

Da die Ökonomie als überwiegendes Betätigungsfeld erscheint, findet eine implizite Reduktion der menschlichen Lebenswelt, der Kultur, auf das Ökonomische statt. Gleichzeitig wird Natur meist selbstverständlich als statisch vorhandenes Naturkapital vorausgesetzt, sie wird ökonomisch verwertet, aber nicht bewertet. So wird bspw. der „Erfolg“ durch erneuerbare Energien und „Energiewende“ hauptsächlich als ökonomischer Erfolg adressiert, während der Anteil von und die Folgen für die Natur ausgeblendet werden (vgl. Kapitel 5.1.2). Dieses Verhältnis zwischen Ökonomie und Natur zeigt sich bereits in den Debatten im Rahmen des Narrativs der ,ökologischen Modernisierung' in der 14. und 15. Legislaturperiode. So sollen unter dem Begriff der ,ökologischen Modernisierung ' Ökologie und Ökonomie zwar vermittelt werden (vgl. z.B. Koalitionsvertrag 1998: 1), aber gleichzeitig wird diese ,Modernisierung“ in erster Linie ökonomischen Zielsetzungen untergeordnet (vgl. Kapitel 5.2.1). Immer wieder scheinen in der Folge teilweise vermittelnde, nicht grundsätzlich dichotome Naturverständnisse auf, bspw. wenn Natur als „Lebensgrundlage“ benannt wird (vgl. z.B. Koalitionsvertrag 2002: 7). Durch den Fokus auf marktorientierte Lösungen werden aber die Produktivitäten und Produktionszyklen der Natur ausgeblendet, Natur wird als passives Objekt marktorientierter Politik abgewertet. Bereits im Begriff, ökologische Modernisierung' spiegeln sich auch die gesellschaftlichen Naturverhältnisse der sogenannten Moderne, in der von Gesellschaft und Natur als getrennten Sphären ausgegangen wird, wider. Die ökologische Krise wird daher nicht als Krisensymptom der Moderne aufgefasst, sondern vielmehr wird von einer Lösung ökologischer Probleme durch ,eine ökologische Modernisierung der Moderne“ ausgegangen, in der Wachstum und Entwicklung ökologisch angepasst werden (Krüger 2015: 112).

Das EEG als politische Praxis im Rahmen des Narrativs ,ökologische Modernisierung' zielt nicht explizit auf eine Organisation erneuerbarer Energien nach marktwirtschaftlichen Prinzipien ab. Es materialisiert sich außerhalb des Bundestags auch in einer nicht-vermarktungsorientierten Vermittlung ${ }^{26}$ von Erhalten und Gestalten, von Schutz und Nutzung, Natur und Kultur. Diese Dimension des EEG bleibt in den Debatten im Bundestag allerdings unbeleuchtet. Im Mittelpunkt steht die Markteinführung von erneuerbaren Energien als Technologie - das EEG wird zum „Marktanreizprogramm“ interpretiert und dem Zweck der „Markteinführung“ erneuerbarer Energien untergeordnet (vgl. Kapitel 5.1.2). Diese werden somit dem ökonomischtechnologischen Bereich zugeordnet (vgl. Kapitel 5.1.2). Die ,automatische‘ ökologische Wirkung der Technologie wird als Annahme mit globalem Geltungsanspruch getroffen: Ohne „Klimaschutz“ mit politischen Inhalten zu füllen, wird gleichzeitig der Eindruck erzeugt, die ökologische Wirkung deutscher Klimapolitik entstehe über den Export erneuerbarer Energien quasi von selbst (vgl. Kapitel 5.2.6). Hier zeigt

25 Eine Ausnahme ist die 16. Legislaturperiode, in der die Debatte um „Energiewende“ aber marginal ist und Kollokationen in diesem Bereich hauptsächlich von der LINKEN gesetzt werden.

26 Bspw. in nicht gewinnorientierten Genossenschaften, durch nicht gewinnmotivierte private Nutzung etc. 
sich deutlich der instrumentelle Aspekt von Externalisierung: Das Abgespaltene wird für die Kapitalverwertung gebraucht.

Die Vorstellung eines dichotomen Verhältnisses zwischen Natur und Ökonomie dominiert die in Verbindung mit „Energiewende“ stehenden Story-Lines: Die Natur wird als das ,Andere' des Marktes betrachtet. Anstatt eine Transformation der Marktökonomie anzustreben, die ein erneuerndes Gestalten durch und mit Natur ermöglicht, wird die Marktökonomie, welche die Natur abspaltet, nicht hinterfragt.

Durch den Fokus auf das technologisch-messbare werden umweltpolitische Inhalte so stark auf Klimaschutz - im Sinne von Reduktion der $\mathrm{CO}_{2}$ Emissionen - reduziert, dass dieser in der 17. Legislaturperiode genutzt werden kann, um für die Laufzeitverlängerung von Atomkraftwerken zu argumentieren (vgl. Kapitel 5.2.1). Durch die Abspaltung sämtlicher ökologischer Folgen von Atomkraft und ihrer Unterordnung unter die Profitrationalität ${ }^{27}$ wird Kernenergie mit dem Ziel des Klimaschutzes artikulatorisch auf dem „Weg in das Zeitalter erneuerbarer Energien“ in einen „dynamischen Energiemix“ integriert (17/3049).

Diese hegemoniale Artikulation erfährt durch „Fukushima“ eine Dislokation: Die ökologischen Folgen der Kernenergie werden sichtbar und somit für kurze Zeit auch die Kultur/Natur Dichotomie. Der Glaube an die technologische Beherrschbarkeit von Natur wird herausgefordert. Der Begriff „Energiewende“ wird nun verwendet, um die Dislokation zu überwinden und die Trennungsstruktur zwischen Natur und Ökonomie wieder zu verdecken (vgl. z.B. CDU 30.06.2011: 13403). Diese Reartikulation in einer Äquivalenzkette mit „Energiewende“ ist jedoch nicht als Internalisierung von Natur zu bewerten, sondern nach Biesecker/von Winterfeld (2014: 14) als „abspaltendes Einbeziehen“ im Rahmen der sich nun ausbreitenden und Schließung verheißenden hegemonialen marktwirtschaftlichen Formation um „Energiewende“. Durch Aufgehen des Narrativs „ökologische Modernisierung“ im fantasmatischen Narrativ der hegemonialen Formation, das ich als technokratisch-managementorientierten Ökokonsens bezeichnet habe, wird Natur nahezu vollständig aus den Dimensionen der Fantasie ausgeschlossen. Ökologische Krisen über den nuklearen Unfall hinaus werden externalisiert und als Gefahr für eine auf Erhalt von Wohlstand ausgerichtete Energiepolitik ausgeblendet (vgl. Kapitel 5.4).

In dieser Weise wird die Natur/Kultur-Dichotomie in den Debatten um „Energiewende“ nach „Fukushima“ nicht nur fortgeschrieben, sie festigt sich sogar: Durch die Artikulation von „Energiewende“ wird die Lösung ökologischer Probleme suggeriert, die aber de facto gar nicht Gegenstand der Debatten sind, sondern permanent aus dem Politischen gedrängt werden.

Der hegemoniale Externalisierungsprozess in Bezug auf die Kultur/Natur Dichotomie kann auch durch das fantasmatische Narrativ ,sozial-ökologischer Umbau“ nicht entscheidend herausgefordert werden, was seiner marginalisierten Diskursposition zuzuschreiben ist. Aufgrund der wenigen Artikulationen, die diesem Narrativ zugeordnet werden konnten, kann darüber hinaus aktuell noch nicht beurteilt werden, ob in ihm die Abspaltung und Abwertung von Natur aus dem Ökonomischen aufgehoben wird.

27 Vgl. z.B. „Wir wollen diesen Lastwagen voller Geld nicht verbrennen“ (CDU 28.10.2010: $7168)$. 


\section{Öffentlich/Privat}

Die Dichotomie öffentlich/privat spielt für die feministische Analyse eine wichtige Rolle, da sie aus der Verknüpfung von Männlichkeit mit Öffentlichkeit und der Verschiebung des Weiblichen ins Private hervorgeht. Das Private wird dabei als Residualkategorie generiert und abgewertet, als das, was das Öffentliche nicht ist. Das Private entspricht also hier dem Externen. Gleichzeitig erfolgt eine implizite hierarchische Bewertung. „Durch ihre Zuordnung zur Privatsphäre [...] werden die Lebensund Arbeitszusammenhänge von Frauen weitgehend unsichtbar gemacht, ihre Erfahrungen, Interessen, Organisations- und Aktionsformen als nicht politikwürdig ausgeschlossen“ (Wischermann 2003: 23).

Wie bereits herausgearbeitet wurde, zeigt sich in den untersuchten Plenardebatten eine Reduktion des Öffentlichen auf das Ökonomische, welche als hauptsächliches Betätigungsfeld erscheint. Die Ökonomie wird zwar der Gesellschaft zugeordnet (soll Arbeitsplätze schaffen etc.), soziale Themen werden aber insbesondere in der Fokussierung auf das Arbeitsplatz-Argument auf den ökonomischen Bereich reduziert (vgl. z.B. Kapitel 5.2.3), nur Erwerbsarbeit wird gesehen. Der Blickes auf das ,Soziale" wird dadurch verengt. Soziale Themen, die sich nicht in der Perspektive auf (Markt)Ökonomie verarbeiten lassen, werden implizit dem privaten Bereich zugeordnet und im Zuge dieses Externalisierungsprozesses kaum artikuliert - so ist von Sorgearbeit keine Rede. Dies wird bereits in der Kollokationsanalyse deutlich, in der sich kaum Differenzbeziehungen zeigen, die auf den sozialen Bereich zielen (vgl. Kapitel 5.1.2). Die Ausgrenzung des Sozialen aus dem sichtbaren öffentlichökonomischen Bereich hat wie oben dargestellt auch eine geschlechtshierarchische Dimension, da der öffentliche Bereich bedingt bspw. durch gesellschaftliche Arbeitsteilung immer noch eher männlich konnotiert ist, während Frauen eher dem privaten Bereich zugeordnet werden, wie die feministische Wissenschaft seit den 1970er Jahren herausgearbeitet hat (vgl.z.B. Bock/Duden 1976; Fraser 1996; Lerner 1991). Damit verwoben sind weitere Dichotomisierungsprozesse: Akteur*innen des öffentlich-ökonomischen Bereichs werden bspw. eher als aktiv konstituiert, Akteur*innen des Privaten eher als passiv (vgl. z.B. die Rolle der Bürger*innen in Kapitel 5.2.8 ).

Die Trennungsstruktur zwischen öffentlich und privat manifestiert sich bereits in den energiepolitischen Debatten im Rahmen des Narrativs ,ökologische Modernisierung' in der 14. und 15. Legislaturperiode. Privatpersonen werden dabei auf ihre Funktion als ökonomische Akteur*innen, meist Konsument*innen oder Investor*innen, reduziert (vgl. Kapitel 5.2.1). In dieser Rolle werden sie zwar in den Bereich des öffentlich-Ökonomischen einbezogen, aber gleichzeitig abspaltend dem Privaten zugeordnet und somit als passiv konstituiert. Die Möglichkeit einer aktiven Konsument*innenfunktion, bei der die Bürger*innen aus eigener Fähigkeit zur Verantwortung heraus nachhaltigen Konsum praktizieren, wird nicht artikuliert. Eine derartiges Handeln aus „Sorge und Vorsorge für sich und für andere“ heraus würde bspw. dem Menschenbild des Vorsorgenden Wirtschaftens entsprechen (Theoriegruppe Vorsorgendes Wirtschaften 2000: 30). Im „Energiewende“ - Diskurs dominieren aber selbst im Narrativ der ,ökologischen Modernisierung ' diskursive Muster, die das Bild von nutzenmaximierenden Konsument*innen zeichnen, die durch veränderte Nachfrage scheinbar, automatisch` auf Marktsignale des öffentlich-ökonomischen Bereichs, wie bspw. Preisänderungen, reagieren. Demnach zielen die als reformistisch klassifizierten politischen Praktiken darauf ab, über marktanreizinitiierte 
Verhaltensänderungen einen Beitrag der Konsument*innen zur ,ökologischen Modernisierung' zu erwirken. Als Beispiel ist die sogenannte Ökosteuer zu nennen, die mit dem Ziel eingeführt wird, „marktwirtschaftliche Anreize für [...] ein umweltbewußtes Verhalten der Verbraucherinnen und Verbraucher" zu setzen (Koalitionsvertrag 1998: 11). Dass die Einnahmen aus der Steuer nicht für ökologische Maßnahmen, sondern für Kosteneinsparungen der Industrie eingesetzt werden (nämlich die Senkung der Lohnnebenkosten (Koalitionsvertrag 1998: 12)), ist als weiteres Indiz für eine Abwertung des Ökologischen und des Sozialen gegenüber dem Marktwirtschaftlichen zu werten. Die sozialen Auswirkungen einer marktorientierten ökologischen Modernisierungspolitik unter dem Motto ,Arbeit verbilligen, Ressourcen verteuern $^{28 ،}$ (SPD 25.11.1999: 6740) werden kaum hinterfragt - die Konsequenzen für den privaten Bereich durch die Verteuerung des Konsums, u.a. für die Sorgearbeit, bleiben überwiegend im Dunkeln. Dem öffentlich-Ökonomischen werden unter dem Primat von Wachstum andere in den Bereich des Privaten verschobene Ziele untergeordnet.

Deutlich wird eine Ausgrenzung des Sozialen aus dem Bereich des öffentlichÖkonomischen auch in den Debatten um die Befreiung der energieintensiven Industrie von der EEG-Umlage in der 16. Legislaturperiode. Während die Auswirkungen des Strompreises auf die Industrie problematisiert werden, werden die Auswirkungen dieser Befreiung auf private Haushalte lediglich von der jeweiligen Opposition adressiert (vgl. Kapitel 5.2.5). Neben ihrer Rolle als Konsument*innen wird den privaten Haushalten spätestens ab der 17. Legislaturperiode hauptsächlich die Aufgabe zugewiesen, die „Energiewende“ zu akzeptieren. Akzeptanz als Ergebnis eines Bewertungsprozesses enthält eine aktive Komponente und birgt über entsprechende Einbeziehung und Beteiligungsmöglichkeiten die Chance einer aktiveren Rolle von Bürger*innen. Schweizer-Ries u.a. (2011: 140ff.) beschreiben eine aktive und eine passive Dimension des Akzeptanzbegriffes, wobei die aktive Dimension aktives Engagement und Unterstützung beinhalte, während die passive Dimension nur auf Befürwortung beruhe. Die in den Debatten um „Energiewende“ adressierte Akzeptanz zielt dabei eher auf einen passiven Akzeptanzbegriff: Bürger*innen sollen die „Energiewende" befürworten und sich nicht dagegen wehren (vgl. z.B. Angela Merkel 09.06.2011: 12961). Dies beinhaltet auch eine fordernde Haltung an die Bürger*innen in einen vermeintlichen energiepolitischen Konsens einzustimmen (vgl. Kapitel 5.2.8), was bspw. beim Thema Netzausbau in den Debatten der 17. und 18. Legislaturperiode eine besondere Rolle spielt (vgl. Kapitel 5.2.7). Dabei wird die mangelnde Akzeptanz als hauptsächlich mitverantwortlich für den zu langsamen Netzausbau dargestellt und die Verantwortung für das langsame Voranschreiten der „Energiewende“ ins Private verschoben, während strukturelle Ursachen wie das Festhalten an konventionellen Kraftwerken trotz massiver Überkapazitäten (www.unendlich-viel-energie.de - verfehlte Ausbauziele) lediglich von der Oppositi$\mathrm{on}^{29}$ thematisiert werden (vgl. Kapitel 5.2.7). Das ins Privaten verschobene Soziale

28 Vgl. z.B.: „Die Kosten für den Faktor Arbeit werden gesenkt. Im Gegenzug wird der Verbrauch von Energie und Rohstoffen verteuert“ (SPD 25.11.1999: 6740).

29 Vgl. z.B.: „Das Falscheste ist, eine neue Fertigung mit 100 Prozent Leistung aufzubauen und die alte Fertigung mit kompletter Kapazität zu erhalten“(LINKE 01.06.2016: 16985). 
erscheint so als Gefahr bzw. Hindernis für die „Energiewende“, während das öffentlich-Ökonomische in Verbindung mit einem technologieorientierten Fortschrittsglauben als wertvoll erscheint.

Politische Willensbekundungen der Bürger*innen scheinen nur erwünscht insofern sie dem vermeintlichen energiepolitischen Konsens entsprechen. Während vor „Fukushima“ einerseits die massiven Proteste von Bürger*innen gegen die Laufzeitverlängerung ignoriert werden (vgl. Kapitel 5.2.8), wird andererseits Akzeptanz für einen „energiepolitischen Konsens“ eingefordert (17/3049: 18). Nach „Fukushima“ werden dagegen eben diese gegen Atomkraft protestierenden Bürger*innen in einen ,neuen Konsens` einbezogen (Angela Merkel 17.03.2011: 10886f.), jedoch ohne ihnen eine aktive Rolle bei der politischen Gestaltung von „Energiewende“ zuzuweisen und ohne ihren Beitrag zu diesem ,neuen Konsens' zu thematisieren. Dieses Einbeziehen ist demnach als ,abspaltendes Einbeziehen` zu bewerten, was sich dadurch verstärkt, dass die Bürger*innen gemeinsam mit der Bundesregierung in ein kollektives „Wir“ eingebunden werden - verbunden mit einem Appell weitere Protestaktionen zu unterlassen (Angela Merkel 09.06.2011: 12961ff.).

Wie sich an den Protesten gegen die Laufzeitverlängerung und vor allem nach „Fukushima“ ablesen lässt, hat die aktive Rolle der Bürger*innen bspw. im Rahmen der Anti-Atombewegung zur Bedeutungsgenerierung von „Energiewende“ im Bundestag zumindest indirekt beigetragen und sich in den folgenden politischen Praktiken auch materialisiert. Die vorliegende Diskursanalyse legt allerdings nahe, dass diese aktive Rolle in den Debatten um „Energiewende“ im Bundestag meist ausgeblendet wird und außerhalb einiger Artikulationen durch die jeweilige Opposition unsichtbar bleibt. Dieser Trennungsprozess, durch den die Bürger*innen aus den politischen Entscheidungen zur „Energiewende“ extrahiert und ins Private verschoben werden, während sich das politische Betätigungsfeld auf das Ökonomische beschränkt, ist Symptom einer sich ausbreitenden hegemonialen marktwirtschaftlichen Formation im „Energiewende“-Diskurs (vgl. Kapitel 5.1.3 und 5.4). Politische Praktiken im Rahmen ,marktorientierter Energiepolitik' folgen der Annahme, dass Innovationen und technische Verbesserungen ausschließlich durch Wettbewerb und Marktanreize (wie Absenkung der Fördersätze) entstehen (CDU 06.06.2008: 17742). Gesellschaftliche Faktoren oder andere Motivationen außerhalb von Gewinnorientierung werden ausgeblendet (vgl. Kapitel 5.3.2). Auch die Bewertung des „Erfolgs“ der „Energiewende“ wird als hauptsächlich abhängig von ökonomischen und technologischen Faktoren konstituiert, während private Haushalte in der Bewertung kaum eine Rolle spielen (vgl. Kollokationsanalyse zu „Erfolg“ in Kapitel 5.1.2). Ihr Anteil am „Erfolg“ der Energiewende bleibt im Dunkeln.

Allerdings geht die Ausbreitung der hegemonialen marktwirtschaftlichen Formation ab der 17. Legislaturperiode auch mit verstärkten Kämpfen um die Zuweisungen zum öffentlichen und privaten Bereich einher. Dies zeigt sich bspw. durch den gestiegenen Signifikanzwert der Kollokationen „Bürgerinnen“ und „Bürger“ in der 18. Legislaturperiode (vgl. Kapitel 5.1.2). Im Zuge der Novellierungen des EEG in der 18. Legislaturperiode droht sich die den Bürger*innen im Diskurs zugeschriebene Rolle in der politischen Praxis weiter zu materialisieren. GRÜNE und LINKE kritisieren, dass durch das veränderte Ausschreibungsdesign hauptsächlich große Konzerne zu Akteur*innen der „Energiewende“ werden (vgl. z.B. LINKE 27.06.2016: 16985). Durch den hohen Aufwand der Teilnahme an Ausschreibungen, seien diese 
für Bürger*innenenergieprojekte, die sich oberhalb einer von Ausschreibungen befreiten Erzeugungsgrenze befinden, kaum noch handhabbar (vgl. Kapitel 5.2.8). Im Zuge des Dissenses über die Rolle der Bürger*innenenergie wird diese verstärkt Gegenstand politischer Praktiken, wobei sie in hegemonialer Aneignung in die marktwirtschaftliche Formation einbezogen wird. ${ }^{30}$ Blieb die Bürger*innenenergie zuvor im Schatten des Privaten, wird sie nun dem Öffentlich-Ökonomischen zugeordnet und droht ohne gleichzeitige Umgestaltung der hier vorherrschenden Machtstrukturen zu verschwinden (vgl. z.B. GRÜNE 01.06.2016: 16974; vgl. Kapitel 5.2.8).

Die Dichotomie öffentlich/privat kann hier auch im Kontext von Geschlecht als Strukturkategorie (Hofmeister u.a. 2013b: 47f.; 62ff.) interpretiert werden, denn es konnte gezeigt werden wie im „Energiewende“-Diskurs die Abgrenzung und Abwertung des Sozialen produziert und reproduziert wird und so die gesellschaftlichen Bedingungsgefüge mit hervorgebracht werden, in denen über die Kategorie Geschlecht als Platzanweiserin das als sozial Weibliches Markierte aus dem Politischen verbannt und ins Private verwiesen wird.

\section{Produktiv/Reproduktiv}

Die Dichotomien öffentlich/privat sowie Natur/Kultur sind mit der Dichotomie produktiv/reproduktiv verwoben: Als produktiv gilt vor dem Hintergrund hegemonialer Gesellschafts- und Naturverhältnisse das öffentlich-Ökonomische, während die dem Privaten zugewiesene als sozial weiblich markierte Produktivität und die Leistungen der Natur als reproduktiv abgespalten werden. Dies manifestiert sich im Rahmen der vorliegenden Diskursanalyse bspw. dadurch, dass der „Energiewende“, bzw. den erneuerbaren Energien, immer dann und nur solange ein hoher Stellenwert beigemessen wird, wie sie aus Perspektive markwirtschaftlicher Diskursmuster als produktiv eingeordnet werden. Energie gilt dabei als „Schlüsselfaktor“ für die Wirtschaft (SPD 27.06.2002: 24776). Ob eine umfassende das Soziale und Ökologische integrierende „Energiewende“ stattfindet oder nicht, erscheint vor dem Hintergrund ,erneuerbarer Energien als Wirtschaftsfaktor` zunächst irrelevant (vgl. Kapitel 5.2.3). Gleichzeitig wir die Produktivität erneuerbarer Energien mythenhaft aufgeladen, mehrfach ist von ,Wundern“ die Rede („Jobwunder“, „Gründungswunder“) (SPD 06.06.2008: 17730). Produktivität wird auf ,warenförmige Arbeit (Erwerbsarbeit) und Kapital“ reduziert (Biesecker/Hofmeister 2006: 133) und nur das wird inwertgesetzt. Die Betonung einer solchen Produktivität erneuerbarer Energien wird in der 17. Legislaturperiode als Begründung herbeigezogen, um den Kurswechsel der schwarz-gelben Regierung nach „Fukushima“ zu legitimieren. Es wird nun von „Energiewende“ gesprochen, während dabei erneuerbare Energien weiterhin überwiegend als Produktionsfaktor adressiert werden. Dessen Inwertsetzung im Rahmen einer „Energiewende“ wird nun auch von CDU, CSU und FDP als internationale „Chance“ gesehen (vgl. FDP 09.06.2011: 12968; vgl. Kapitel 5.2.3).

30 Vgl. z.B.: „Es ist doch so, dass die Fakten insoweit eindeutig sind, dass [...] wir jetzt an einem Punkt sind, an dem die erneuerbaren Energien den Systemwechsel schaffen müssen und auch schaffen werden, nämlich von dem bewährten System des EEG hin zu einem System mit Wettbewerb und Marktwirtschaft“ (CDU 01.06.2016: 16990). 
Das vermeintlich Reproduktive, die sozial weibliche Produktivität und die Naturproduktivität, wird vom vermeintlich Produktiven abgespalten und kommt als Element in den Debatten kaum vor - die Ökonomie erscheint als „Durchflussökonomie“ (Biesecker/Hofmeister 2006: 133). Marktorientierte Instrumente wie der Emissionshandel nehmen zwar Naturleistungen zum Teil in den Blick und scheinen die Dichotomien zwischen Be- und Verwerten sowie Schutz und Nutzung teilweise zu überwinden, indem sie $\mathrm{CO}_{2}$ bewerten. Allerdings wird auch dabei das lineare Modell der „Durchflussökonomie“ nicht durchbrochen, da sich der Preis an Angebot und Nachfrage bemisst und die naturale Produktion wiederum ausgeblendet wird. Es wird davon ausgegangen, dass der Emissionshandel automatisch zu „Umweltschutz“ führe (CDU 28.05.2004: 10236) ohne das Verhältnis von Preis zum Aufwand naturaler (Re)Produktion zu betrachten. Dabei bieten die erneuerbaren Energien die Chance, Naturproduktivität sichtbar zu machen, da sich die Hybridität von erneuerbarer Energie als ,NaturKulturProdukt‘ (vgl. Biesecker/Hofmeister 2006: 147ff.) aufdrängt. Diese Hybridität scheint im Diskurs aber nur an wenigen Stellen durch („Denn die Sonne schickt uns keine Rechnung" (GRUENE 28.05.2004: 10245)“). Gleichzeitig wird scheinbar von einer unendlichen Ressourcenverfügbarkeit ausgegangen (vgl. z.B. SPD 25.08.2004: 10235), wodurch der Anteil der ,Natur' an der Produktion der Solarmodule oder anderer Anlagen, und verbunden damit die Endlichkeit der verfügbaren Ressourcen, ausgeblendet werden.

Ebenso selten wie Naturproduktivität wird sozial weibliche Produktivität adressiert, wie bspw. die Produktivität der ins Private verwiesenen Sorgearbeit. Private energieverbrauchende vermeintliche Konsumtion kommt in ihren produktiven Tätigkeitsformen (bspw. in Form von Wäsche waschen oder Kochen) nicht in den Blick und wird als passive Handlung beschrieben, die durch Preissignale gesteuert werde (s.o., Ökosteuer). Gleichzeitig wird ein großer Teil ökologischer Verantwortung in diesen privaten Konsumbereich verschoben, da staatliche Eingriffe in den marktwirtschaftlichen Produktionsbereich unerwünscht sind und mitunter sogar zur wesentlichen Gefahr für und gleichzeitig durch die „Energiewende“ stilisiert werden. Vor diesem Hintergrund wird im Diskurs an die „Eigenverantwortung“ und „Vernunft“ der Konsument*innen appelliert. ${ }^{31}$

Mit der diskursiven Ausgrenzung der Produktivität vermeintlicher Konsumtion, zum Beispiel in Form einer aktiven auf „Sorge und Vorsorge für sich und für andere“ (Theoriegruppe Vorsorgendes Wirtschaften 2000: 30) ausgerichteten Konsument*innenrolle, geht das Ausblenden der Produktivität des Privaten im Energiebereich einher. Der aktive Anteil, den Haushalte sowohl am Energieverbrauch als auch der -produktion innehaben, wird größtenteils nicht thematisiert, obwohl die „Energiewende" Chancen bietet, die Dichotomie zwischen Produktion und vermeintlicher Konsumtion auf Haushaltsebene zu überwinden, indem die Haushalte nicht mehr nur scheinbar passive Konsument*innen sondern auch Produzent*innen von Energie sein können (Prosumer*innen). Auch auf diese Prosumtion zeichnet sich aber eine sozial

31 Vgl. z.B.: „Dabei setzt die Bundesregierung auf Vernunft und Eigenverantwortung von Wirtschaft und Bürgern und nicht auf mehr Bürokratie“ ... „Unternehmer und [...] Verbraucher“ sollen „bisher ungenutzte Potentiale im Bereich Energieeffizienz aus eigenem Antrieb“ erschließen (17/3049: 6). 
männliche Perspektive ab, indem als Produzent dann der „Familienvater“ genannt wird (GRÜNE 29.03.2012)). Eine mögliche Prosument*innenfunktion der Haushalte wird insgesamt aber kaum in den Blick genommen. ${ }^{32}$ Ein Aufbrechen der Dichotomie produktiv/reproduktiv ließe sich bspw. anhand von Modellen angehen, bei denen Mieter*innen an der Stromproduktion beteiligt sind. Tatsächlich können Mieter*innen durch Einführung des Mieterstromgesetzes 2017 stärker und aktiver an der Konsumtion von erneuerbarem Strom teilhaben - sie können selbst entscheiden, ob sie den Strom aus der häuslichen Solaranlage beziehen oder nicht. Allerdings haben sie meist keinen Anteil an der Stromproduktion, die in der Hand des*r Hausbesitzer*in bleibt (vgl. Kapitel 5.2.8). ${ }^{33}$ Vom Mieterstromgesetz profitieren aufgrund der gesetzlichen Bedingungen - neben den Mieter*innen ${ }^{34}$ - hauptsächlich Hauseigentümer*innen, die bereits zahlreiche Wohneinheiten besitzen sowie Energieversorgungsunternehmen, die die Anlagen betreiben, wenn der bürokratische Aufwand für den*die Vermieter*in zu hoch ist.

Weitere Chancen für eine aktive Konsument*innenrolle im Rahmen der „Energiewende“ werden im Diskurs gar nicht erst abgebildet. So könnten bspw. Haushalte innerhalb von sogenannten Microgrids ${ }^{35}$ auf intelligente Weise so verknüpft werden, dass nicht die Nachfrage an ein zentrales Angebot angepasst werden müsste, also sich der private Stromverbrauch nach dem öffentlichen Angebot richten müsste, sondern stattdessen eine beiderseitige Anpassung auf lokaler Ebene erfolgen würde. Nachbar*innen könnten untereinander innerhalb ihrer lokalen Gemeinschaften mit Strom handeln, wodurch auch Konsument*innen ohne eigene Solaranlage lokal produzierten Strom erwerben könnten und so mehr Entscheidungsmacht erhielten (Mengelkamp u.a. 2018: 207f.). Stattdessen soll in der politischen Praxis die Anpassung aber zukünftig über ,nachfrageseitiges Lastmanagament“ innerhalb von weiter zentral gesteuerten „Smart Grids“ erfolgen (17/3049:11). Über „Anreize in den Stromtarifen" sollen Konsument*innen ihre Nachfrage an das zentrale Angebot anpassen (17/3049:11). Zwar erhielten Konsument*innen eine begrenzte Entscheidungsmacht, ihren Stromverbrauch zeitlich an die Stromproduktion anzupassen, diese Anpassung wäre aber einseitig nachfragebasiert und würde über sogenannte ,Smart Meters' zentral gesteuert. Es wäre also eine Anpassung der Lebensgewohnheiten und somit des vermeintlich reproduktiven Bereiches an die Stromproduktion notwendig, was die hierarchische Strukturierung der Dichotomie produktiv/reproduk-

32 Die Buchstabenfolge Prosumer* taucht im gesamten Korpus in Bezug auf Energie lediglich drei Mal auf.

33 Darüber hinaus kommen laut einer Studie des BMWi aufgrund verschiedener Bedingungen nur maximal ca. 18\% der Mietwohnungen, insgesamt 3,8 Millionen Haushalte, für Mieterstrom in Frage (Koepp u.a. 2017: 82f.). Bspw. lohnt sich Mieterstrom nur bei einer höheren Anzahl von Wohnungen innerhalb eines Hauses. Weitere Einflussfaktoren sind der hohe bürokratische Aufwand, Sanierungsstand, Baujahr und Eigentumsverhältnisse (ebenda).

34 Die Strompreise für Mieterstrom müssen laut Gesetz um mindestens 10\% niedriger sein als ortsübliche Strompreise.

35 Microgrids sind Kleinststromnetze, die in der Regel mit dem zentralen Netz verbunden sind, aber auch autark funktionieren. 
tiv und somit eine Aneignung des vermeintlich Reproduktiven durch das vermeintlich Produktive erneut reproduzieren würde.

Ebenso wie die Dichotomie öffentlich/privat kann auch die Dichotomie produktiv/reproduktiv im Kontext von Geschlecht als Strukturkategorie (Hofmeister u.a. 2013b: 47f.; 62ff. ) interpretiert werden. Es konnte gezeigt werden wie im „Energiewende"-Diskurs im Bundestag die Abspaltung des vermeintlich Reproduktiven diskursiv reproduziert wird, indem dieses vermeintlich Reproduktive kein Gegenstand politischer Debatten ist und somit die Voraussetzungen für dessen Aneignung innerhalb politischer Praktiken geschaffen werden - die Auswirkungen dieser politischen Praktiken auf das Abgespaltene werden nicht betrachtet. Dementsprechend werden vorherrschende strukturelle, geschlechtlich kodierte Machtverhältnisse reproduziert.

\section{Zentral/Dezentral}

In der sich außerhalb des Bundestags materialisierenden „Energiewende“ zeigen sich veränderte gesellschaftliche Naturverhältnisse vor allem auch mit Blick auf die räumlichen Strukturen. „So werden die im fossilen Energiesystem dominanten zentralen Raumbeziehungen tendenziell durch dezentrale Produktionsstrukturen ersetzt“ (Amri-Henkel u.a. 2017: 16). Während eine zentrale Organisation der Energieversorgung weitgehend in den Händen privater großer Unternehmen liegt (also dem öffentlichen Bereich zugeordnet ist), ist die Akteur*innenvielfalt bei einer dezentralen Organisation wesentlich größer, da vermehrt kleinere Produzent*innen wie private Haushalte eine Rolle spielen. Die zentrale Organisation des Energiesystems bleibt in den politischen Debatten allerdings weitgehend unhinterfragt, liegt implizit zu Grunde, während die materiell bereits stattfindende Dezentralisierung durch erneuerbare Energien im Dunkeln bleibt. Die Kollokation „dezentral“ spielt für die Bedeutungsgenerierung von „Energiewende“ kaum eine Rolle und taucht zum ersten Mal in der 18. Legislaturperiode auf. Sie wird allerdings ausschließlich von der Opposition verwendet und bleibt dadurch in ihrer Bedeutung marginal (vgl. Kapitel 5.1.2). Auch der Ausbau der Netzinfrastruktur (vgl. Kapitel 5.2.7) erfolgt unter der Annahme großer Stromerzeugungszentren auf See und in Küstenregionen, ist also eher auf eine zukünftige zentrale Organisation ausgerichtet. Dies spiegelt sich entsprechend in den Artikulationen im Diskurs wider. Potenziale für eine Dezentralisierung durch die „Energiewende“ werden dadurch verdeckt und ins Außen des „Energiewende“Diskurses verwiesen.

\section{Rational/Emotional - Objektiv/ldeologisch}

Die Binarisierung von Gefühl und Vernunft sowie deren geschlechtliche Kodierung ist ein zentraler Aspekt der Geschlechterforschung. Geschlechterdiskurs und Gefühlsdiskurs können als zeitgleich grundlegend für die Herausbildung des bürgerlichkapitalistischen Staates und für die patriarchale Herrschaft betrachtet werden. Im kapitalistischen Rationalisierungsprozess werden nur bestimmte als männlich kodierte Emotionen als nützlich und berechtigt gewertet, und somit als produktiv konstituiert ${ }^{36}$

36 „Die Kontrolle von Leidenschaften und ihre Kanalisation in Interessen ist Teil eines Gefühlsdispositivs, mit dem sich das Bürgertum beste Bedingungen zu seiner Reproduktion schuf. Das Gefühlsdispositiv ist insofern »produktiv« und nicht zuvörderst repressiv, weil 
- der Rest werde der Natur und den Frauen zugewiesen (Sauer 1997: 9f.). Die geschlechtliche Kodierung des bürgerlichen Gefühlsdiskurses hat auch eine räumliche Dimension, indem weibliche Emotionen in den privaten Bereich verschoben werden, während Rationalität als vermeintlich männliche Eigenschaft sachliches, regelgeleitetes Entscheiden ermöglicht und dem öffentlichen Raum zugewiesen wird (ebenda: 10). (Sozial weibliche) Emotionalität wird zum ,Außen` des Politischen, sie wird als Gefahr für den politischen Raum konstituiert. Dieser Dichotomisierungsprozess zwischen Rationalität und Emotionalität zeigt sich deutlich in den Debatten zur „Energiewende“. Dabei wird Rationalität als handlungsleitendes Prinzip mit Objektivität verbunden, während politische Positionen der Gegner*innen als ideologisch, irrational und vermeintlich emotional abgewertet werden (vgl. Kapitel 5.4) - die Dichotomie rational/emotional wird auf diese Weise mit der Dichotomie objektiv/ideologisch verbunden. Vermeintlich rationale politische Positionen werden so naturalisiert, während andere, davon abweichende Positionen, als unsachlich gewertet werden. Gleichzeitig wird Vernunft, also Rationalität, im Sinne des bürgerlich-kapitalistischen Verwertungsprozesses auf marktökonomische ,Vernunft ‘ reduziert - ohne dies explizit zu machen. Als vernünftig gilt, was gut für die Wirtschaft ist (vgl. z.B. SPD 09.09. 2004: 11256; vgl. Kapitel 5.2.3). Diese Dichotomisierungsprozesse zwischen rational und emotional sowie objektiv und ideologisch zeigen sich besonders deutlich anhand der Debatte um die Laufzeitverlängerung der 17. Legislaturperiode, schreiben sich aber auch nach „Fukushima“ fort (vgl. Kapitel 5.4). Durch die Äquivalenzierung von ,wirtschaftlicher Vernunft', ,Ideologiefreiheit', ,Marktorientierung' und ,Technologieoffenheit“ (17/3050; 17/3049) vor „Fukushima“" entsteht eine antagonistische Zweiteilung des diskursiven Raumes. Das antagonistische Außen, das ,Andere“, bleibt unbenannt, wird aber implizit als ,emotional', ,unsachlich', ,irrational' und ,unwirtschaftlich' abgewertet. Eine Energiepolitik ohne Kernenergie wird so zunächst implizit diesem ,Anderen' zugeordnet, wird zum ,ideologischen“ und ,parteipolitische[n] Kampfthema“ erklärt (CDU 28.10.2010: 7179). „[W]irtschaftliche Vernunft", die CDU, CSU und FDP den anderen Fraktionen absprechen, sei Voraussetzung für die Erreichung ökologischer Ziele (FDP 28.10.2010: 7187).

In Kapitel 5.4 wurde gezeigt wie die Dislokation „Fukushima“ mit der Aneignung von „Energiewende“ durch eine sich ausbreitende hegemoniale marktwirtschaftliche Formation um den ,technologisch-managementorientierten Ökokonsens überwunden wurde: Die „Energiewende“ wird nicht mehr diskursiv im Außen verortet, sondern wird vor dem Hintergrund einer ,neuen Lage‘ in die Äquivalenzkette um ,Vernunft' und ,Objektivität‘ integriert und nun ihrerseits von ,Emotionalität‘ und ,Ideologie" abgegrenzt. Gleichzeitig wird die Ursache für die Katastrophe in „Fukushima“" als nicht objektiv vorhersehbar konstruiert (Angela Merkel 17.03.2011: 10884f.). Die „Emotionalität dieser Debatte“ nach „Fukushima“ sei daher nicht gerechtfertigt (FDP 17.03.2011: 10895), die Opposition sei „hysterisch“, wobei „hysterisch“ im genannten Beispiel explizit auf eine weibliche Vorrednerin abzielt, um de-

es zunächst einmal die regulierte »leidenschaftslose« Realisierung von ökonomischen Interessen in einem »gefühlsfreien« Raum erlaubte“. Gier, Habsucht und Gewinnsucht waren Emotionen, die im Kapitalisierungsprozess nutzbringend eingesetzt werden konnten (Sauer 1997: 9). 
ren Beitrag gegenüber der als ,Norm“ deklarierten ,Vernunft‘ abzuwerten. ${ }^{37}$ Die „Energiewende“ als Teil des ,neuen Konsenses“ solle dabei in Abgrenzung zu früheren Ansätzen der rot-grünen Bundesregierung, die „nur ihre Ideologie befriedigt“ habe, „vernünftig[...]“ sein (FDP 09.06.2011: 12967). Rationalität als vermeintliche ,Entpersönlichung، von Herrschaft solle die Berechenbarkeit politischer Handlungsabläufe suggerieren (Sauer 1997: 12): Während die Opposition von ,glauben“ spreche und „,von der Realität [...] wenig Ahnung“ habe, rede die CDU von „Fakten“ (CDU 29.03.2012: 20304). Der Prozess einer vermeintlichen „Versachlichung“ der „Energiewende“ erscheint hier als „Störfreimachung“ (vgl. Sauer 1997: 12) durch Zurückdrängung nicht-marktwirtschaftlicher Positionen und Emotionen. Politische Positionen, die nicht mit einem Verständnis von „Energiewende“ unter der StoryLine ,Marktorientierung als Bedingung von Energiepolitik' kompatibel sind, werden dem ,Anderen' zugewiesen und als ,ideologisch', ,unwirtschaftlich', ,irrational', ,emotional‘ oder ,sozialistisch“ abgespalten und abgewertet ${ }^{38}$ (vgl. Kapitel 5.4). Untermauert wird das Postulat einer vermeintlich objektiven Sicht auf die „Energiewende" durch die vermehrte Einberufung von Expert*innenkommissionen - beginnend mit der ,Ethikkommission Sichere Energieversorgung‘. Letztlich wird dies zur Legitimation politischer Entscheidungen angeführt- ohne, dass auf die Ergebnisse etwaiger Kommissionen zwangsläufig politische Konsequenzen folgen müssen (vgl. Kapitel 5.2.9).

Sieht sich die SPD noch in der 17. Legislaturperiode selbst mit dem Ideologievorwurf konfrontiert, ändert sie in der Regierungskoalition der 18. Legislaturperiode ihre Diskursposition und wertet die Kritik der Opposition selbst als ,irrational', ,ideologisch“ (SPD 01.06.2016: 16979) und ,emotional“ (,aufgeregt“ (SPD 10.04.2014: 2443)), bzw. „schrill ${ }^{39 ،}$ ab. Ein „nüchterneres“ im Gegensatz zu einem ,emotionalen“ Verhältnis zur „Energiewende“ („Die Euphorie ist verflogen“) wertet die CDU gegen Ende der 18. Legislaturperiode als positiv für die „deutsche Volkswirtschaft“ (CDU 29.06.2017 24992). Emotionalität erscheint hier als Störfaktor für die „Energiewende“, eine Gefahr für die politische Debatte über sie und ihre marktwirtschaftliche Entwicklung.

Analog zur Dichotomie rational/emotional zeigten sich auch die Dichotomien öffentlich/privat sowie produktiv/reproduktiv analytisch fruchtbar, um die Geschlech-

37 „Es ist klug, in diesem Zusammenhang nicht panikhaft und hysterisch zu agieren, sondern die Dinge sachlich und richtig zu analysieren und dann vernünftig zu entscheiden. [...] Frau Höhn, mit den Worten ,panikhaft" und ,hysterisch“ habe ich auch Sie gemeint" (CSU 24.03.2011: 11300).

38 Vgl. z.B.: „Bei den ideologischen Ergüssen und sozialistischen Ausarbeitungen Ihrerseits fällt es manchmal schwer, gelassen zu bleiben, [...]. Meine Damen und Herren, ich weiß, dass Sie Probleme mit dem demokratischen Rechtsstaat und dem demokratischen System haben. Aber nicht Sie entscheiden über Recht und Unrecht; [...] Darüber entscheidet auch kein ZK oder Politbüro, sondern dieses Haus [...]. Meine Damen und Herren, wir haben es nicht nötig, uns mit solchen Leuten auf Anti-Marktwirtschafts-Debatten einzulassen“ (CDU 29.03.2012: 20292f).

39 Vgl. z.B.: „Gott sei Dank kümmert sich die Energiewende nicht um solche schrillen Reden“ (SPD 01.06.2016: 16981). 
terperspektive auf „Energiewende“ zu vervollständigen. Diese Externalisierungsprozesse stehen für eine Ausgrenzung des dem weiblichen zugeschriebenen Sozialen aus dem öffentlichen Politischen in Verbindung mit einer Abwertung des als reproduktiv abgewerteten Sozialen und Ökologischen. Eine Vermittlung zwischen ,Innen“ und ,Außen` im Rahmen der Kategorie der (Re)Produktivität findet nicht statt. Wie bereits dargestellt zeigt sich in diesen Dichotomien Geschlecht als gesellschaftliche Strukturkategorie. Darüber hinaus kann insbesondere die Dichotomie rational/ emotional auch im Kontext von Geschlecht als Prozesskategorie (,doing gender ${ }^{6}$ ) interpretiert werden. ,Doing gender' fragt danach wie Geschlecht in sozialen Prozessen reproduziert und hervorgebracht, also hergestellt wird (West/Zimmermann 1987). Mit der Abwertung speziell weiblicher Rednerinnen als ,hysterisch', wird nicht nur die Bedeutung wiederholt, die Emotionalität als Ausdruck weiblicher Natur festschreibt, sondern gleichzeitig auch das soziale Arrangement gerechtfertigt, welches eben diese Emotionalität aus dem politischen Raum verbannt. Die Selbstverständlichkeit, mit der eine vermeintliche Sachlichkeit/Rationalität als einzig legitimer Modus politischer Debatten festgeschrieben wird, verdeckt dessen (sozial männliche) Konstruktion und verunmöglicht die Frage nach dem geschlechtlich kodierten Herstellungsmodus dieser Aufteilung (vgl. auch Gildemeister 2008: 137).

\section{Natur- und Technikwissenschaft/Geisteswissenschaft}

Die Dichotomien Natur/Kultur sowie öffentlich/privat spiegeln sich auch in einer Spaltung der Wissenschaft in Naturwissenschaften auf der einen und Geisteswissenschaften auf der anderen Seite, die nicht nur methodisch, sondern auch institutionell abgegrenzt sind, wider. „Sie scheinen sich auf getrennte Bereiche in der realen Welt zu beziehen: auf die anorganische und die organische Natur einerseits, auf Kultur und Gesellschaft andererseits“ (Becker/Jahn 2006: 110). Die Geisteswissenschaften werden dabei gegenüber den Naturwissenschaften, die als objektiv gelten, abgewertet. Analog zu den bisher bereits analysierten Dichotomien, spielen in den Debatten zur „Energiewende“ Natur- und Technikwissenschaften eine deutlich größere Rolle als Sozialwissenschaften. Dabei wird eine Verwertungsorientierung der Wissenschaft deutlich $^{40}$, vor deren Hintergrund die Sozialwissenschaften aus dem Blickfeld geraten (vgl. Kapitel 5.2.9). Während Ergebnisse sozialwissenschaftlicher Forschung - mit Ausnahme der Akzeptanzforschung - häufig nicht direkt als marktökonomisch verwertbar in Betracht gezogen werden, wird die Erforschung der Natur und der Technik als Grundlage für die Ausbeutung der Natur herangezogen, durch die Akkumulation also erst möglich wird. Technologische Energieforschung - personifiziert durch den ,deutschen Ingenieur “ - solle Garant „für Wachstum und Wohlstand" sein und dem Export dienen (CDU 13.06.2013: 31547; CDU 30.06.2011: 13369).

Gesellschaftswissenschaftliche Themen, die zum Beispiel auf soziale Folgen des Klimawandels abzielen, werden wie gezeigt im Diskurs größtenteils überhaupt nicht benannt, die integrativen Potenziale sozial-ökologischer Forschung werden nicht

40 Vgl. z.B.: „Forschungsergebnisse“ zur „Energiewende“ sollen ,zügig in Form von innovativen und marktfähigen Produkten verwertet werden“ müssen (Koalitionsvertrag 2013: 25f.). 
gesehen. Umweltprobleme werden im Hinblick auf ihre technische Lösbarkeit betrachtet, während ihre sozialen und ökonomischen Ursachen und Wirkungen unbenannt bleiben. Die GRÜNEN kritisieren daher eine mangelnde interdisziplinäre Ausrichtung (GRÜNE 13.06.2013: 31550f.), die LINKEN fordern eine Ausweitung der Energieforschung zur Transformationsforschung (LINKE 13.06.2013: 31549) (vgl. Kapitel 5.2.9). Diese Forderungen bleiben marginalisiert, da sich auch in der Bezugnahme auf die Wissenschaft das Narrativ des ,technokratisch-managementorientierten Ökokonsenses` als Teil der hegemonialen marktwirtschaftlichen Diskursformation durchsetzt.

\section{Neokoloniale und nationale Externalisierung als Prinzip im Diskurs um „Energiewende“}

Wie in 3.2.2 dargelegt, zeigte sich Externalisierung als Prinzip im Zuge der Analyse deutlich auch in Form nationaler und neokolonialer Externalisierung, sodass eine Erweiterung der Theorie und Methodik notwendig wurde. Neokoloniale und nationale Externalisierung als Prinzip verweisen darüber hinaus auf die Kultur/Natur Dichotomie sowie die Verwobenheit von Geschlechterverhältnissen mit globalen rassistischen Ausbeutungsstrukturen und anderen intersektionalen Ausschließungsprozessen, wie im Folgenden deutlich gemacht wird.

Im vorliegenden Unterkapitel zeige ich anhand der in Kapitel 3.2.2 dargestellten Theorie, wie sich Formen des ,Othering sowie der Erzeugung von Subalternität, auch im Querschnitt mit hegemonialem Wissen und (neo)kolonialen Vorstellungen von Zeitlichkeit, in den energiepolitischen Debatten manifestieren. Darüber hinaus wird die Bedeutung der mit nationalen Motiven aufgeladenen Story-Line ,Deutschland als Vorreiter' im Zusammenhang mit energiepolitischen Praktiken und der Entwicklung des „Energiewende“ - Diskurses insgesamt analysiert.

Im Diskurs über „Energiewende“ wird das ,Andere“ auf vielfältige Weise produziert. ,Othering' manifestiert sich in den energiepolitischen Debatten ab der 14. Legislaturperiode unter anderem in der Dimension des ,Worldings' (des Weltenmachens). Dabei steht zum einen die Konsolidierung eines ,deutschen Selbst‘ im Mittelpunkt. So wird die Einführung erneuerbarer Energien von Anfang an mit einer deutschen Sonderrolle und einer internationalen Vorbildfunktion verknüpft (vgl. Kapitel 5.2.6). Zum anderen werden , andere‘ Länder, allen voran sogenannte Entwicklungsländer, von diesem konsolidierten Selbst als ,Vorbild‘ ausgehend gleichzeitig als unterlegen konstituiert. Es wird der Anschein erweckt, dass sie darauf angewiesen seien, sich am deutschen Vorbild zu orientieren und sie scheinbar die deutsche „Energiewende“ geradezu hoffnungsvoll erwarten. ${ }^{41}$ Dieses ebenso nationale wie (neo)koloniale diskursive Muster begründet sich auf einem ,Worlding“ in Verbindung mit der hegemonialen westlichen Wissensordnung. So wird im Rahmen der Story-Line ,Deutschland als Vorreiter' das Wissen über eine nachhaltige Energiepolitik als etwas ,Eigenes“ konstituiert, während die ,Anderen“ dieses Wissen mehr oder weniger passiv übernehmen. Diese Perspektive findet beispielsweise im Selbst-

41 Vgl. z.B.: „Ich bin mir ganz sicher, dass viele internationale Augen auf dieses Gesetz schauen werden und viele Erwartungen und Hoffnungen damit verbunden sind" (SPD 25.02.2000: 8437). 
verständnis deutscher Parlamentarier im Hinblick auf renewabales2004 Konferenz ihren Ausdruck. Durch ihr Wissen über die „Energiewende“ sprachen sie sich im Vorfeld der Konferenz die Befähigung zu, den ,Anderen“ „die Augen zu öffnen“, „zu zeigen, was wirklich möglich ist“ (SPD 28.05.2004: 10240).

Im nächsten Schritt zeigt sich in der Story-Line ,Deutschland als Vorreiter ‘ der Anspruch auf eine Globalisierung der „Energiewende“, wobei der Fokus dieser Globalisierung implizit auf die sogenannten Entwicklungsländern gelegt wird. Hier zeigt sich die Überschneidung von Wissen und Macht, bzw. was nach Spivak (1985: 255f.) als „epistemische Gewalt“ bezeichnet werden kann: „Entwicklungsländer dürfen und wollen nicht die Fehler wiederholen, die die Industrieländer bisher bei ihrer Energieversorgung gemacht haben, denn das hält unser Globus nicht aus [...]“ (SPD 28.05. 2004: 10234). Deutlich wird hier auch eine paternalistische Haltung: Der vermeintliche Wissensvorsprung wird nicht nur als Legitimation dafür herangezogen, sogenannten Entwicklungsländern die Art und Weise ihrer Entwicklung vorzuschreiben, ohne dabei die tatsächlich zu diesem Zeitpunkt existierende eigene Energieversorgung zu hinterfragen ${ }^{42}$, er soll auch als Grundlage dienen für eine Beurteilung darüber, welche Energieversorgung für die ,anderen' Länder am besten $\mathrm{sei}^{43}$, ohne deren Bedürfnisse anzuhören. Der erneuerbare Energiebereich ermögliche eine gemeinsame Lösung von ,globalen Entwicklungsproblemen und globalen Umweltproblemen“ (SPD 28.05.2004: 10241). Eine deutsche „Energiewende“, die physisch-materiell faktisch zu diesem Zeitpunkt kaum vorhanden ist, wird international zur Norm erhoben.

Hier wird zum einen augenscheinlich, was Bhabha (1994: 142) als disjunktive Temporalität der Moderne bezeichnet (vgl. Kapitel 3.2.2) - nämlich das Ausblenden der gewaltvollen Ursprünge dieser sogenannten ,Entwicklungsprobleme', die sich in neokolonialen Machtverhältnissen weiter fortschreiben, bei gleichzeitiger Betonung eines westlichen Fortschritts- und Rationalitätsnarrativs (Fortschritt durch westliche Technologie erneuerbarer Energien). Zum anderen zeigt sich auch die von Spivak (1985) beschriebene Kompliz*innenschaft zwischen westlicher Wissensformation und Wirtschaftsinteressen (Castro Varela/Dhawan 2015: 193): Denn aus der ,eigenen' Erkenntnis über Fortschrittspotenziale für Entwicklungsländer durch erneuerbare Energien, wird direkt die Notwendigkeit abgeleitet, dort vermehrt zu ,investieren“ (GRÜNE 28.05.2004: 10238). Dabei wird weder benannt, dass die Artikulation von ,Investitionen“ überwiegend auf Kredite sowie auf Exportförderung abzielt, noch werden Abhängigkeitsverhältnisse, die damit in Verbindung stehen, reflektiert. Es wird sogar eine Verantwortung konstruiert, die Deutschland als „Exportland“ zu tragen habe, um die erneuerbaren Energien „dort“ - „,in der Dritten Welt“ - „,wirksam werden zu lassen“" (SPD 25.02.2000: 8438). Investitionen, die sich in ihrem materiellen Gehalt überwiegend als Kredite oder Zinssubventionen darstellen, werden als fi-

42 So wird beispielsweise der Eindruck vermittelt, als sei die „Energiewende“ in Deutschland bereits ,vollzogen“ (GRÜNE 28.05.2004: 10237). Dagegen betrug der Anteil erneuerbarer Energien am Primärenergieverbrauch zu diesem Zeitpunkt (2004) lediglich 4,5\% und am Bruttostromverbrauch 9,4\% (www.erneuerbare-energien.de).

43 Vgl. z.B.: „Außerdem bringt der Einsatz erneuerbarer Energien riesige Vorteile für Entwicklungsländer mit sich“ (SPD 28.05.2004: 10234). 
nanzielle „Zusagen“ konstruiert (SPD 02.07.2009: 25698) und erscheinen so als vermeintlich bedingungslose Hilfe, die mit einer Aufwertung der eigenen moralischen Position verbunden wird. ${ }^{44}$ Während das ,Eigene' also als technologisch fortschrittlich, verantwortungsbewusst und darüber hinaus als moralisch konsolidiert wird, erscheint das ,Andere` von dieser Position des Selbst aus als hilfsbedürftig, rückständig, nicht eigenständig, sondern von „deutscher Spitzentechnologie“ (CDU 02.07. 2009: 25690) abhängig ${ }^{45}$ und gleichzeitig räumlich betrachtet weit entfernt („,dort“ (SPD 25.02.2000: 8438)). Das ,Eigene“ hilft - dem ,Anderen“ wird geholfen.

So wird gleichzeitig das Zeichen der ,Dritten Welt‘ vernebelt (vgl. Kapitel 3.2.2), indem die machtvolle Herstellung dieser Einteilung der Welt nicht benannt wird, während über Exportförderung und Kreditvergaben neokoloniale Abhängigkeitsverhältnisse gestärkt werden. Über die moralische Rechtfertigung von Exportförderung und insbesondere ab der 17. Legislaturperiode auch von Rohstoffsicherung (17/3049: 18) durch Herstellung eines Zusammenhangs mit Entwicklungszusammenarbeit werden Wirtschaftsinteressen verdeckt ${ }^{46}$ oder legitimiert. ${ }^{47}$ Dabei ist es vor allem die vermeintliche Attraktivität aus marktwirtschaftlicher Perspektive, die von Anfang an zu einer breiten Zustimmung zur Förderung des Exportes erneuerbarer Energien in Verbindung mit Entwicklungszusammenarbeit führt (vgl. Kapitel 5.2.6). ${ }^{48} \mathrm{Im} \mathrm{Zu}$ sammenhang zwischen Wissensordnung und Wirtschaftsinteressen, wie er sich in der Exportförderung zeigt, wird es darüber hinaus erforderlich, Wissen als das ,Eigene“ zu reproduzieren und zu erhalten. Das technologische Wissen wird selbst als Kapital im Wirtschaftsprozess betrachtet. Dagegen fordert die LINKE „energietechnologisches Wissen mit den Ländern des Südens, zum Beispiel den Ländern Afrikas, vorbehaltlos“ zu teilen (LINKE 02.07.2009: 25695). ${ }^{49}$

44 Vgl. z.B.: „In dem vorgesehenen Zeitraum von 2003 bis 2007 wurden in der bilateralen Zusammenarbeit insgesamt 1,3 Milliarden Euro für erneuerbare Energien zugesagt. Ich finde, das kann sich sehen lassen“ (SPD 02.07.2009: 25698).

45 Vgl. z.B.: „Mit den Exportinitiativen „Erneuerbare Energien“ und „Energieeffizienz“ unterstützt die Bundesregierung gezielt deutsche Unternehmen und die weltweite Verbreitung deutscher Spitzentechnologie, gerade auch in Entwicklungs- und Schwellenländern“ (CDU 02.07.2009: 25690).

46 Vgl. z.B.: „Unser politikfeldübergreifender Ansatz bedeutet nach unserem Verständnis nämlich nicht, dass Entwicklungspolitik Mittel zum Zweck wird“ (SPD 02.07.2009: 25697).

47 Vgl. z.B.: „Energieaußenpolitik ist vor allem auch Entwicklungspolitik“ und „EnergieauBenpolitik insbesondere auch Wirtschaftsförderung und Wirtschaftspolitik“ (CDU 02.07. 2009: 25697).

48 So argumentiert die CSU Deutschland solle die Technologie „dort einsetzen, wo sie viel mehr und viel effizienter zum Klimaschutz beitragen kann [...]“ (CSU 25.02.2000: 8440) und die FDP möchte ,in Entwicklungs- und Schwellenländern [...] investieren, weil dort eine Reduktion der Emissionen von $\mathrm{CO}_{2}$ zu deutlich geringeren Kosten möglich ist“ und so „Technologietransfer“ und „Exportoffensive“ verbunden werden können (FDP 28.05.2004: 10238).

49 In dieser Forderung wird die Beanspruchung des technologischen Wissenskapitals für das ,Selbst‘ zwar überwunden, die Zuordnung des Wissens zum ,Eigenen` bleibt aber erhalten. 
Wie sich hier bereits andeutet, zeigt sich in den Debatten zur Energiepolitik nicht nur die Konsolidierung des eigenen deutschen Selbst als das ,Normale', von dem aus das ,Andere“ der sogenannten Entwicklungsländer als unterlegen (,Worlding') konstituiert wird. Die Subjekte des ,Anderen“ werden auch gleichzeitig als statisch, passiv und moralisch unterlegen herabgewürdigt (zweite Dimension des ,Othering'). Dies wird beispielsweise in der Rolle, die den , anderen` Parlamentarier*innen bei der renewables2004 Konferenz zugewiesen wird, deutlich: Sie kommen nach Bonn, „um diese in Deutschland vollzogene Energiewende zu besichtigen" (GRÜNE 28.05. 2004: 10237). Die Parlamentarier*innen aus anderen Staaten, vor allem aus sogenannten Entwicklungsländern, erscheinen dabei als passiv, unentwickelt, rückstän$\operatorname{dig}^{50}$ aber beeinfluss- und formbar (ihnen werden somit vermeintlich weibliche Eigenschaften zugeschrieben) - während die als aktiv und rational geltenden deutschen Parlamentarier*innen scheinbar von einem besonders privilegierten Punkt aus die Welt erklären und zur Nachahmung ermutigen. ${ }^{51}$ Es zeigt sich deutlich, wie die westliche hegemoniale Wissensordnung das Selbst- und Fremdbild prägt. Vor dem Hintergrund der Zuweisung von Technologie und Wissen zum ,Eigenen', erscheint das ,Andere“ als vermeintlich schwach, im Gegensatz zum starken ,Eigenen“: „Der Entwicklungsminister Gerd Müller hat [...] den Startschuss für das Projekt ,Grüne Bürgerenergie für Afrika' gegeben. [...] Ein Solarunternehmer aus Kamerun hat auf diesem Kongress gesprochen. Der Schlusssatz seiner Rede war: Für uns ist alles, was aus Deutschland kommt, stark" (CDU 29.06.2017: 24992). Dabei zeigt sich auch, wie diese Abwertungsstruktur für die deutsche Volkswirtschaft nutzbar gemacht werden kann. ${ }^{52}$

Vor dem Hintergrund der Annahme dieser hegemonialen Wissensordnung wird der ,deutsche Ingenieur' zur Personifikation von Produktivität, Wissen und Rationalität erhoben. Nicht nur Bewohner*innen von sogenannten Entwicklungsländern werden zur Antithese dieser Eigenschaften angeführt, sondern sie wird auch auf sogenannte ,orientalische' Länder übertragen, deren wirtschaftliche Entscheidungsträger als unkontrollierbar oder gar ,verrückt“ konstituiert werden: „Lieber deutsche Ingenieure als verrückte Derwische in Nahost als Garanten unserer Energieversorgung!“ (FDP 09.09.2004: 11229). Zudem wird das Wort ,Basar' als Metapher für vermeintliche Ineffizienz und Unkontrollierbarkeit orientalischer Märkte im Gegen-

50 Vgl. z.B.: „Ich habe einen ziemlich guten Überblick über das, was in den Ländern auf den verschiedenen Kontinenten passiert. In Deutschland sind die Koalitionsparteien in dieser Frage deutlich weiter als die Oppositionsparteien. Die Oppositionsparteien sind in dieser Frage aber durchaus weiter als manche sozialdemokratischen Parteien in anderen Ländern“ (SPD 28.05.2004: 10242).

51 Vgl. z.B.: „Wir wollen die anderen Parlamentarier motivieren, positive Beispiele nachzuahmen, sie ermutigen, die Initiativen auch von sich aus zu ergreifen“ (SPD 28.05.2004: 10240).

$52 \mathrm{Zu}$ der Bemerkung des Solarunternehmers aus Kamerun fügt die CDU hinzu: „Wir haben ein derart großes Vertrauenskapital bei den erneuerbaren Energien und der Energiewende, das es für unsere Volkswirtschaft zu nutzen gilt“" (CDU 29.06.2017: 24992). 
satz zur westlichen Marktökonomie bemüht. ${ }^{53}$ Sowohl bei ,Basar` als auch bei ,Derwisch` handelt es sich um generalisierende Repräsentationen des ,Orients` (Said 1979: 231), die hier dazu verwendet werden, um gegnerische politische Positionen abzuwerten. Das orientalische Stereotyp dient hier analog zu Said (1979: 7) dem vermeintlich vernunftgeleiteten und hoch entwickelten ,deutschen Selbst ${ }^{6}$ als sein minderwertiges Gegenbild.

Anhand des durch die CDU angeführten Beispiels des Solarunternehmers aus Kamerun, lässt sich im Ansatz nachvollziehen, wie Subalternität ${ }^{54}$ als Position der radikalen Differenz zum westlichen ,Selbst' produziert wird. Während der als männlich gekennzeichnete Solarunternehmer als Mitglied einer vermeintlichen ,Elite“ seines Landes immerhin indirekt adressiert wird, werden subalterne Frauen oder Angehörige ,unterer Klassen', Menschen die in keiner Weise Zugang zu einer herrschenden ,Elite“ haben, in den Debatten nicht erwähnt. Gleichzeitig bewegt sich selbst das diskursiv erzeugte Bild des Solarunternehmers innerhalb der westlichen Wissensordnung, die seinen Status im Diskurs und die Bewertung seines Handelns determiniert und nach der ihm keine Möglichkeit ,autonom` zu wissen und somit zu sprechen zugestanden wird. Elemente von Subalternität tauchen auch in der Personifikation des ,verrückten Derwischs“ als männlicher Repräsentant einer ,fremden“ ,orientalischen“ ,Elite` auf. Er wird antithetisch dem männlichen „deutschen Ingenieur“ gegenüber gestellt. Alles dazwischen verbleibt im Dunkeln: Auf der einen Seite die nicht sozial männlichen, nicht-technologieorientierten Beiträge zu einer deutschen Energieversorgung, die im hegemonial männlichen Diskurs ebenfalls abgespalten werden. Auf der anderen Seite subalterne Subjekte (z.B. Frauen), die der ohnehin bereits abgewerteten männlichen Position der ,orientalischen“ ,Elite" noch weiter untergeordnet werden. Die Orientalität überschreibt dabei jede andere Eigenschaft: „An Oriental man was first an Oriental and only second a man“ (Said 1979: 231). Rassismus, Sexismus und Klassismus wirken ineinander.

Die Bedeutung des „Energiewende“-Diskurses für die Konsolidierung eines ,deutschen Selbst', und umgekehrt das Bemühen nationaler narrativer Muster zur Begründung der "Energiewende“ nach „Fukushima“ (vgl. Kapitel 5.4), ist vollumfänglich erst im Laufe der Diskursanalyse augenscheinlich geworden. Während der Auswertung des Datenmaterials ergab sich daher die Notwendigkeit, die Verknüpfungen zwischen erneuerbaren Energien und „Energiewende“ mit Erzählungen über Nation genauer zu betrachten, was wie beschrieben auch eine theoretische Erweiterung des Kriterienrahmens des Vorsorgenden Wirtschaftens und eine entsprechende Anpassung der Analyseheuristik erforderte. Mithilfe dieser Erweiterung lassen sich Kontinuitäten der europäischen Aufklärung in Verbindung mit nationalen und neokolonialen Motiven in den energiepolitischen Debatten im Bundestag nachzeichnen,

53 Vgl. z.B. die Bemerkung der FDP, die Preisaufsicht sei „,nichts anderes als ineffiziente Basarökonomie“, die für den Wettbewerb eine „Katastrophe“ wäre und Arbeitsplätze „,vom Markt [...]feg[e]“ (FDP 02.07.2009: 25875).

54 Um Subalternität im Diskurs um „Energiewende“ genauer zu untersuchen, sind weitere Forschungen nötig. Hier wäre es sicher notwendig nicht nur Debatten aus dem deutschen Parlament zu betrachten, sondern auch internationale Dokumente/Vorgänge, bspw. aus der Entwicklungszusammenarbeit. 
z.B. anhand der Annahme ,der Privilegierung des bürgerlichen männlichen Subjekts als universelles Subjekt der Vernunft" (Castro Varela/Dhawan 2015: 202) gegenüber dem ,Anderen“, wie sie bspw. in der Gegenüberstellung von ,deutschem Ingenieur und ,verrücktem Derwisch' (s.o.) projiziert wird. Im Rahmen der Story-Line ,Deutschland als Vorreiter' werden ,deutsche' Technologie und das damit verbundene Wissen zu erneuerbaren Energien als „Symbole eines wünschenswerten Fortschritts“ (Castro Varela/Dhawan 2015: 38) gedeutet (s.o.). Im „Energiewende“ Diskurs erscheinen dabei ,Fortschritt' und ,Rationalität' als Produkte deutscher Aufklärung. Um diese Verknüpfung aufrechtzuerhalten, wird zum einen jeglicher $\mathrm{Zu}$ sammenhang mit (neo)kolonialen Machtverhältnissen ausgeblendet und zum anderen die Umsetzung der „Energiewende“ innerhalb Deutschlands diskursiv mit einem gewissen ,Sendungsbewusstsein“ verbunden. ${ }^{55}$ Es wird das Bild von „denen“ gezeichnet, die ,unserem“, deutschen Vorbild“ folgen und dadurch Entwicklung erfahren (Castro Varela/Dhawan 2015: 87). ${ }^{56}$

Infolge der Debatten um die renewables2004 Konferenz verstetigt sich die StoryLine ,Deutschland als Vorreiter“ im Verlauf des „Energiewende“-Diskurses als Moment nationaler Identität, das schließlich auch den konservativen und liberalen Fraktionen einen positiven Bezug auf das EEG ermöglicht. ${ }^{57}$ Die ,Entwicklungsprobleme“ (s.o.) , anderer' Länder werden als Mangel konstruiert und gleichzeitig als Legitimation für Deutschland, globale Verantwortung durch Export zu übernehmen, herangezogen. Das Vordringen deutscher Unternehmen und deutscher Spitzentechnologie in die ,unentwickelte“ Welt wird so (erneut) als „Triumph der Wissenschaft und Rationalität" (Castro Varela/Dhawan 2015: 38) und somit der Aufklärung über das Unwissen stilisiert:

„Nun ist es unsere Aufgabe, Impulse zu setzen. Im Rahmen dieser Konferenz müssen wir auf-
zeigen, welche Möglichkeiten die erneuerbaren Energien tatsächlich bieten. Für deren Einsatz
gibt es nicht nur Umweltgründe [...] sondern auch entwicklungspolitische Gründe. Viele Län-
der der Dritten Welt sitzen mittlerweile in der Falle der fossilen Energien [...]. Hier liegt die

55 Vgl. z.B.: „Wir sind mit der Energiewende angetreten, um zu zeigen [...], dass eine hochentwickelte Volkswirtschaft wie die deutsche es schafft, sich von nuklearer, langfristig aber auch von fossiler Energieversorgung zu befreien, ohne dabei ihren wirtschaftlichen Erfolg zu gefährden“ (SPD 27.06.3014: 3932). Vgl. z.B.: „Die ganze Welt schaut staunend auf Deutschland, wo sich in kürzester Zeit eine [...] industrielle Entwicklung für Solarzellen, Windkraft und Biogas entwickelt hat" (GRÜNE 06.06.2008: 17737).

56 Dieser Zusammenhang wird ab der 16. Legislaturperiode hauptsächlich von den LINKEN und teilweise auch von den GRÜNEN herausgefordert, deren Positionen aber marginalisiert bleiben. Bspw. heben die GRÜNEN hervor, dass die „entwickelten Länder immer noch über 50 oder 60 Prozent des Gases und Öles für 15 Prozent der Weltbevölkerung beanspruchen“ (GRÜNE 02.07.2009: 25689). Die LINKEN heben die strategischen Interessen in der internationalen Zusammenarbeit hervor: „Die Bundesregierung betreibt Aufrüstung, weil sie fossile Energiequellen und die Transportwege sichern will“ (LINKE 02.07.2009: 25694).

57 Vgl. z.B.: „Das deutsche EEG gilt in der Welt als Modell und Vorbild dafür, wie man erneuerbare Energien im Strombereich fördern kann“ (CDU 06.06.2008: 17742). 
große Chance durch erneuerbare Energien [...]. Wir sollten stolz darauf sein, hierzu eine avantgardistische Position einnehmen zu können“ (SPD 28.05.2004: 10241).

Die Story-Line ,Deutschland als Vorreiter' wird dabei eng mit dem Motiv ,Deutschland als Exportnation“ im Bereich erneuerbarer Energien verknüpft (vgl. z.B. FDP 09.06.2011: 12968), was eine Konsolidierung kapitalistischer Interessen im Kontext einer nationalen Erzählung von „Energiewende“ zeigt.

Die wichtige Rolle ,erneuerbarer Energien“ in der Konstruktion nationaler Identität, zeigt sich auch anhand der Debatte um die Laufzeitverlängerung 2010: So betont die Regierungskoalition dabei ebenfalls die internationale Vorbildfunktion Deutschlands ${ }^{58}$ im Bereich erneuerbarer Energien, während die Verlängerung der Laufzeiten der Kernkraftwerke als ,Brücke“ artikuliert wird. Es ist also nicht die „Energiewen$\mathrm{de}^{\text {" }}$ als Idee, sondern insbesondere die erneuerbaren Energien als Technologie, die bereits vor „Fukushima“ auch aufgrund ihrer Funktion für die nationale Identitätskonstruktion breite Anerkennung findet. Nach „Fukushima“ wird „Energiewende“ in die Konstruktion nationaler Identität integriert. Dies wird auch durch die signifikante Verbindung von „Energiewende“ und „Deutschland“ ab der 17. Legislaturperiode deutlich (vgl. Kapitel 5.1.2). Dabei ermöglichen die bereits zuvor mit erneuerbaren Energien verknüpften Bilder deutscher ,Stärke' und einer deutschen ,Vorbildfunktion“ die Aneignung des Begriffs „Energiewende“ für die nationale Erzählung in besonderer Weise: „Wenn es ein Land schaffen kann, dann ist es Deutschland“ (CDU 30.06.2011: 13370). ${ }^{59}$ Die deutsche „Energiewende“ wird zum internationalen „Leitprojekt für den Klimaschutz" erklärt, an dem sich andere Länder orientieren (FDP 13.06.2013: 31292). Erneut wird diese ,Vorbildfunktion“ auch mit einer internationalen moralischen Verantwortung verbunden. ${ }^{60}$ Durch die Berufung auf eine konstruierte nationale Vergangenheit werden die Risse in der Gegenwart verdeckt (vgl. Bhabha 1994: 142), die durch die mit „Fukushima“ zu Tage geförderten Inkohärenz ${ }^{61}$ sichtbar wurden. Besonders deutlich zeigt sich dies in Angela Merkels Regierungserklärung zum Atomausstieg im Juni 2011:

„Deutschland hat schon so manches Mal gezeigt, was es kann, was in ihm steckt, und hat schon ganz andere Herausforderungen bewältigt: die Einführung der sozialen Marktwirtschaft, weltweit in dieser Form einmalig; die Vollendung der deutschen Einheit, historisch ohne Vorbild; aus der weltweiten Finanz- und Wirtschaftskrise stärker herausgekommen, als wir in sie hineingegangen sind, und $-\mathrm{ja}$, auch das - besser als die meisten anderen [...]“ (Angela Merkel 09.06.2011: 12963f.).

58 Vgl. z.B.: „Wir sind dort weltweit an der Spitze und einzigartig“ (CDU 28.10.2010: 7168).

59 Vgl. z.B.: „Welches Land, wenn nicht unser Land, sollte dazu die Kraft haben?“ (Angela Merkel 09.06.2011: 12963f.).

60 Vgl. z.B.: „Jetzt schaut die Welt auf uns [...]. Wir haben die Verantwortung, zu zeigen, dass der Umbau funktioniert“ (GRÜNE 30.06.2011: 13381).

61 Bspw. das Sichtbarwerden einer nicht-nachhaltigen, möglicherweise nicht-vorbildhaften, da auf Atomenergie basierenden Energieversorgung. 
Die diskursive Verknüpfung von „Energiewende“ mit der Erzählung von Nation trägt dazu bei, die Kohärenz im energiepolitischen Diskurs nach „Fukushima“ zumindest scheinbar wiederherzustellen. So wird die neue identitäre Erzählung von „Energiewende“ auch zur Erzählung von nationaler Gemeinschaft und Kultur: „Wir sind das Land, das für neue Technik, Pioniergeist und höchste Ingenieurkunst steht. Wir sind das Land der Ideen, das Zukunftsvisionen mit Ernsthaftigkeit, Genauigkeit und Verantwortung für zukünftige Generationen Wirklichkeit werden lässt [...]“ (Angela Merkel 09.06.2011: 12963f.). Die Nation erscheint hier zeitlos und eins. Die Ambivalenzen, die sich durch „Fukushima“ zeigten und das Konfliktive in den historischen Entscheidungen zur Atomenergie offenbarten, werden durch die Berufung auf eine nationale Gemeinschaft verdeckt. ${ }^{62}$ Dabei wird die Performanz, die Veränderbarkeit der Erzählung der Nation (vgl. Bhabha 1990; Castro Varela/Dhawan 2015: 257f.) deutlich: Bei Konfrontation mit mangelnder Kohärenz eignet sich die nationale Identität neue Erzählungen (hier: die „Energiewende“) an, wodurch sich Ausschließungsprozesse verschieben. Das Beharren der Erzählung der Nation auf Gleichzeitigkeit, wird u.a. daran sichtbar, dass die Regierungskoalition die Entscheidung zur Atomenergie als Fehler kollektiviert, in die Vergangenheit verschiebt, ihren eigenen politischen Anteil leugnet und die Entscheidung damit entpolitisiert: „In den 60er-Jahren ist ein kollektiver Fehler begangen worden - übrigens im Einvernehmen der großen Parteien -, nämlich die Entscheidung, maßgeblich auf Atomenergie zu setzen“ (CDU 30.06.2011: 13383). ${ }^{63}$ Die politische Auseinandersetzung mit diesen Fehlern wird dann durch den Verweis dies sei eine „Historikerdebatte“, man solle sich „stärker dem Ausblick als dem Rückblick widmen“ (CSU 30.06.2011: 13392) delegitimiert. Die nationale Gemeinschaft als Basis energiepolitischer Entscheidungen kann nur auf der Grundlage des Dementierens von Heterogenität und Uneinigkeit konstruiert werden. Sie beruht damit parasitär auf dem Ausschluss von Vielfalt. Es wird an ein nationales ,Wir' (vgl. Kapitel 5.2.6) als Basis einer nationalen Einigkeit und Einstimmigkeit appelliert:

„Aber man muss auch ein Gespür dafür haben, dass es jetzt nicht nur darum geht, recht zu haben und in einzelnen Punkten auf seiner Meinung zu bestehen [...], sondern man muss auch

62 Auch die Entscheidung zur „Energiewende“ erscheint nun als Verdienst der nationalen Gemeinschaft, während der Anteil von zivilgesellschaftlichen Protesten wie der AntiAtombewegung verdeckt wird. Die LINKE hebt dies hervor: „Warum - das ist doch eine spannende Frage - gelingt das zuerst in Deutschland und nicht in Frankreich oder Polen? Ich kann Ihnen sagen, warum: Weil es in Deutschland eine ungeheuer starke Antiatombewegung gibt, die jetzt einen Erfolg feiert, für den sie jahrzehntelang gekämpft hat“ (LINKE 09.06.2011: 12969).

63 Interessant ist hier, dass eine Politisierung dieses Fehlers (hier in Bezug auf eine Rede des Abgeordneten der LINKEN Gregor Gysi) direkt im nächsten Satz durch eine Abwertung der Kritik daran delegitimiert wird: „Herr Gysi, Sie und Ihr System hatten mit kollektiven Irrtümern ja viel zu tun; denn Sie haben immerhin 40 Jahre lang versucht, darauf eine Republik zu gründen [...]. Insofern war es sehr beeindruckend, wie Sie sich hier hingestellt haben“(CDU 30.06.2011: 13383). 
begreifen, dass jetzt dieses nationale Werk in Deutschland losgeht [...]. Sie sollten jetzt endgültig über Ihren Schatten springen“ (CDU 30.06.2011: 13368).

In der Erzählung von nationaler Gemeinschaft und Kultur sowie im Appell an Einigkeit zeigt sich deutlich die pädagogische Dimension des nationalen Narrativs: Die Historizität der Nation wird behauptet, aber ihre Ambivalenz gleichzeitig verdrängt die Konfliktivität in der Geschichtlichkeit von „Energiewende“, die sich im Zusammenhang mit den Protesten gegen Atomkraft und für erneuerbare Energien seit den 70er Jahren und nach „Fukushima“ sowie gegen die Laufzeitverlängerung 2010 zeigt, wird durch die Berufung auf eine nationale Einheit verdeckt. So erscheint die „Energiewende“ im Bundestag letztlich als enthistorisierte „Energiewende“.

Im Zuge der nationalen und neokolonialen Externalisierungsprozesse, die sich über den „Energiewende“-Diskurs hinweg entfalten, lassen sich wie auch anhand der zuvor analysierten Dichotomien die hegemoniale Gesellschafts- und Naturverhältnisse nachzeichnen. Die Auslagerung von Sozialem und Ökologischem aus dem unter rein ökonomischen Kriterien betrachteten Politischen wird durch die Konstruktion nationaler Identität weiter verstärkt und in ihrer neokolonialen Dimension vertieft. Insgesamt wurde deutlich, dass in Bezug auf die aufgezeigten Dichotomien in der hegemonialen Diskursformation keine aussichtsreichen Ansätze für Vermittlung bspw. im Sinne der Kategorie der (Re)Produktivität aufgezeigt werden.

\subsection{POTENZIALE FÜR EINE HERRSCHAFTSÄRMERE ZUKUNFTSGESTALTUNG IM DISKURS UM ,ENERGIEWENDE“}

In Kapitel 3.2.2 wurde ,Potenziale für eine herrschaftsärmere Zukunftsgestaltung ‘ als weiteres Kriterium Vorsorgenden Wirtschaftens eingeführt. Es fragt in dieser Arbeit vor allem nach den Potenzialen, die sich im energiepolitischen Diskurs im Bundestag für eine herrschaftsärmere Zukunftsgestaltung zeigen, wozu es in Hinblick auf den Umgang mit Externalisierungen bereits im vorausgehenden Kapitel erste Rückschlüsse gab. Dies wird nun mit der Frage nach Gerechtigkeit ${ }^{64}$ - als Legitimation von politischen Praktiken, die auf eine herrschaftsärmere Zukunftsgestaltung zielen im Kontext des Ansatzes von ,Affirmation“ und ,Transformation' nach Fraser (2001: 47ff.) sowie der Frage nach Eigentum (Biesecker/von Winterfeld 2011) verbunden.

In Kapitel 5.3.1 wurden die politischen Praktiken, die sich im Kontext des fantasmatischen Narrativs, ökologische Modernisierung' zeigen, als reformistisch ausgerichtet klassifiziert, jedoch wurde wegen einer strukturell externen Verortung der Hindernisse/Gefahren auf eine beharrende Wirkung bezüglich ökonomischer Strukturen verwiesen. Im Rahmen der in Kapitel 5.3.2 erfolgten Klassifizierung wurden politischen Praktiken im Kontext des Narrativs ,marktwirtschaftliche Energiepolitik sowohl in Bezug auf die externe Gefahrenverortung als auch auf die am Status-Quo orientierte Glücksverheißung eine beharrende Ausrichtung attestiert und reformisti-

64 Für den begrifflichen Umgang mit ,Gerechtigkeit` in der vorliegenden Arbeit vgl. Fußnote 43 in Kapitel 3. 